\title{
La violencia en el Perú 2015 \\ Violence in Peru 2015
}

\begin{abstract}
Fausto Garmendia ${ }^{1,2}$
'Médico-Cirujano, Doctor en Medicina; Instituto de Investigaciones Clinicas "Fausto Garmendia Lorena", Programa Permanente de Capacitación para la Atención Integral de las Víctimas de la Violencia, Facultad de Medicina, Universidad Nacional Mayor de San Marcos,

${ }^{2}$ Académico Honorario, Academia Nacional de Medicina
\end{abstract}

\begin{abstract}
Resumen
Se efectúa una revisión de los tipos más frecuentes de violencia que se producen actualmente en el Perú, desde la política, autoinflingida, interpersonal dentro de la familia, producida a la mujer, infanto-juvenil y adultos mayores. Se hace una referencia histórica a la Divina Comedia de Dante Alighieri en cuanto a sus contenidos sobre la violencia y sus perspectivas hacia la actualidad. Se destacan las formas emergentes de violencia de tipo económico, delincuencial, laboral y ecológica. Se propone una nueva clasificación de la violencia tomando en cuenta las nuevas modalidades. Se discute el modelo ecológico para explicar las raíces de la violencia y finalmente se sugieren acciones y prácticas para amenguar la prevalencia de la violencia en el Perú.
\end{abstract}

Palabras clave. Violencia, emergente y reemergente; Perú.

Abstract

The most frequent types of violence occurring currently in Peru, including the political, self-inflicted, interpersonal within the family, produced to women, adolescents and older adults are reviewed. A historical reference to Dante Alighieri's Divine Comedy is made as to its contents on violence and its perspectives for today. It highlights emerging forms of economic, criminal, labor and ecological types of violence. A new classification of violence taking into account the new modalities is proposed. The ecological model to explain the roots of violence is discussed and finally actions and practices are suggested for lessening the prevalence of violence in Peru.

Keywords. Violence, emergent and reemergent; Peru.

An Fac med. 2016;77(2):153-61/ http://dx.doi.org/10.15381/anales.v77i2.11838

\section{INTRODUCCIÓN}

La toma de conciencia sobre la violencia como problema social y de salud en el Perú se inició a partir del conflicto interno terrorista de 1980 al 2000, cuando dos grupos de extrema izquierda, Sendero Luminoso (SL) y el Movimiento Revolucionario Túpac Amaru (MRTA) irrumpieron en el escenario nacional desconociendo el orden constitucional establecido, utilizando diversas formas de intimidación y muerte, tales como acciones armadas, genocidios, sabotajes, aniquilamientos selectivos, masacres, torturas, exilio forzado, explosión de bombas, apagones por destrucción de torres de alta tensión eléctrica, paros armados, cautiverio, esclavización de comunidades e intimidación colectiva, entre otros.

Finalizado el conflicto en el año 2000 por la acción de las Fuerzas Armadas, que también cometieron excesos, en el gobierno transitorio presidido por el Dr. Valentín Paniagua Corazao, se formó la denominada Comisión de la Verdad y Reconciliación (CVR) para estudiar e informar sobre la magnitud y consecuencias de las dos décadas de actividad terrorista. El 28 de agosto del 2003, la CVR hizo entrega oficial del Informe Final de sus actividades al gobierno del Dr. Alejandro Toledo ${ }^{(1)}$.
La CVR formuló un Plan Integral de Reparaciones, que fijaba como objetivo general "Reparar y compensar la violación de los derechos humanos, así como las pérdidas y daños sociales, morales y materiales sufridos por las víctimas como resultado del conflicto armado interno", organizado en seis programas complementarios no excluyentes, consistente en reparaciones simbólicas, reparaciones en salud, reparaciones en educación, restitución de derechos, reparaciones económicas y reparaciones colectivas.

El objetivo de las Reparaciones en Salud estuvo orientado a la recuperación de la salud mental y física, recons- 
titución de las redes de soporte social y el fortalecimiento de las capacidades para el desarrollo personal y social. Las Líneas de Acción comprendían: capacitación, recuperación integral desde la intervención comunitaria, recuperación integral desde la intervención clínica, acceso a la salud y promoción de la salud

Con la finalidad de instrumentar las reparaciones en salud y en educación, el gobierno a través del primer ministro Dr. Luis Solari de la Fuente, en el año 2003 convocó a la Facultad de Medicina de la UNMSM. En nuestra condición de Decano de la Facultad de Medicina y por decisión unánime del Consejo de Facultad, se aceptó el reto y se creó el Programa Permanente de Capacitación para la Atención Integral de las Víctimas de la Violencia (PPCAI$\mathrm{VV})$, con lo que nuestra institución se comprometió desde entonces a trabajar en este problema, fundamentalmente en la formación y capacitación de recursos humanos para la atención integral de las víctimas de violencia.

\section{VIOLENCIA POLÍTICA}

La CVR señaló que el conflicto había cobrado 69280 muertes y desapariciones, $75 \%$ de los cuales eran hablantes de lenguas nativas, $79 \%$ campesinos, $56 \%$ dedicados a actividades agropecuarias, $68 \%$ con educación primaria o menor; quedaron 20000 viudas, 40000 huérfanos, 435 comunidades fueron arrasadas, sinnúmero de desplazados, 1,5 a 2 millones de afectados directamente y, al final, mayor pobreza.

La actividad del PPCAIVV, sin embargo, no se ha limitado a la violencia política, sino también a otros tipos de violencia que se incrementaron a partir de la época del terrorismo. En este trabajo, se hará inicialmente referencia a las formas de violencia tratadas con mayor frecuencia, como la autoinflingida, la familiar ejercida principalmente contra las mujeres, los niños y al adulto mayor, para luego destacar otras formas de violencia emergentes que se han iniciado o intensificado en el Perú en años recientes, con el objetivo de proveer una visión global y actualizada de todos los tipos de violencia que se están produciendo en el Perú. Con esta finalidad es, así mismo, necesario recordar la definición de violencia propugnada por la OMS como "El uso deliberado de la fuerza física o el poder, ya sea en grado de amenaza o efectivo, contra uno mismo, otra persona o un grupo o comunidad, que cause o tenga muchas probabilidades de causar lesiones, muerte, daños psicológicos, trastornos del desarrollo o privaciones.

\section{VIOLENCIA AUTOINFLINGIDA}

De acuerdo a la Sociedad Peruana de Prevención del Suicidio, en el Perú se ha registrado una prevalencia de 305 a 334 suicidios anuales entre los años 2012 a $2014^{(2)}$, cifras a las cuales se debería agregar los intentos de suicidio y lo que se denomina conducta suicida ${ }^{(3)}$.

\section{VIOLENCIA CONTRA LA MUJER}

La violencia contra la mujer es muy alta en el Perú, en particular la violen- cia psicológica que se encuentra en el rango del $70 \%$ y que, como se puede ver en la gráfica 1 , no ha variado significativamente desde el año $2009{ }^{(4)}$.

Este tipo de agresión es expresada a través de palabras, gestos o ademanes y que consisten en injurias, calumnias, gritos, insultos, desprecios, burlas, ironías, situaciones de control de la libertad individual, humillaciones, amenazas y otras orientadas a socavar la autoestima.

En el año 2000, la violencia física a nivel nacional alcanzaba el 41,2\%, con una distribución regional que fluctuaba entre $29 \%$ y $57,3 \%$. En el año 2014 , el promedio se redujo a $32,3 \%$, con una distribución regional diferente que varió entre $22,7 \%$ y $46,7 \%$ que, en parte, parece tener relación con la influencia que tuvo la violencia política de los años 1980-2000; es decir, en las regiones donde la violencia política fue mayor el promedio es mayor que en aquellas que fue menor (ver tabla 1).

Las denuncias por violencia sexual a mujeres mayores de 18 años ha fluctuado entre 1200 a 1600 por año (gráfica 2) en los años 2011 a 2013, la gran mayoría en el ámbito de Lima ${ }^{(7)}$. Las cifras por cierto deben ser mayores, desde que

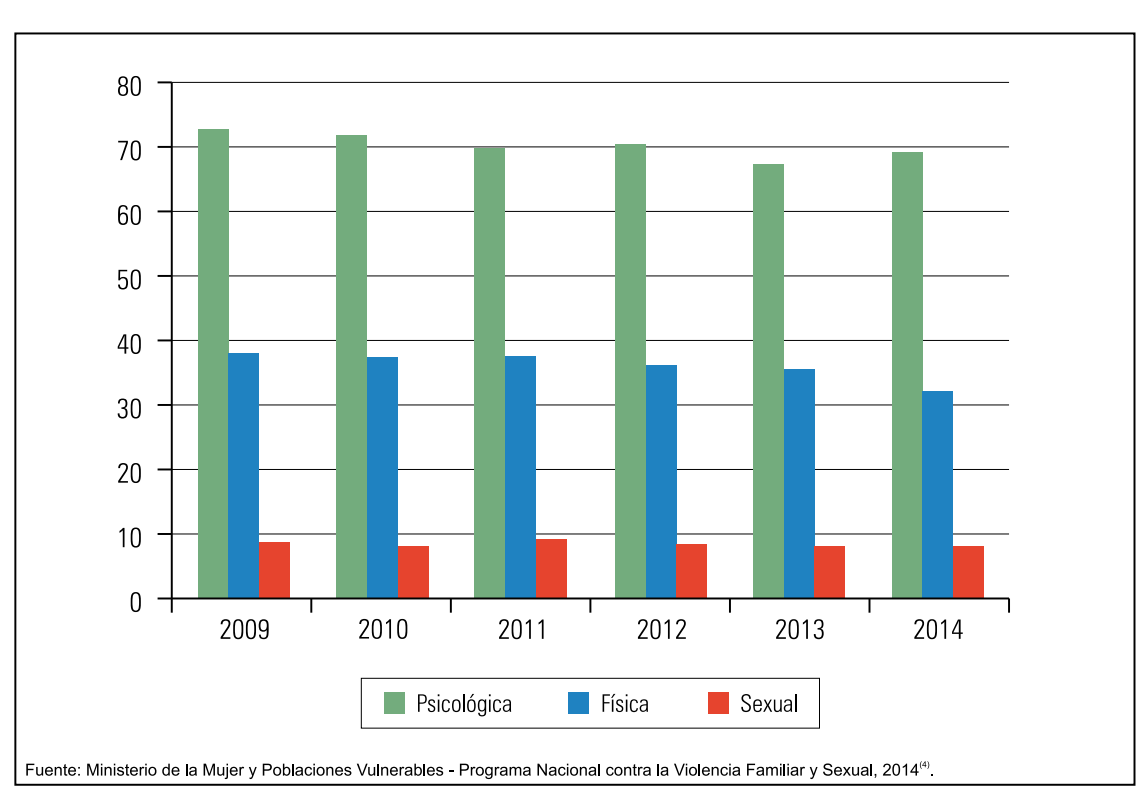

Gráfica 1. Violencia contra la mujer a nivel nacional. 
Tabla 1. Relación de la violencia física contra la mujer con la violencia política (1980-2000).

\begin{tabular}{ccc} 
Ámbito & $\begin{array}{c}\text { ENDES 2000, } \\
\text { porcentaje }\end{array}$ & $\begin{array}{c}\text { ENDES 2014, } \\
\text { porcentaje }\end{array}$ \\
Nacional & 42,1 & 32,3 \\
Regiones con poca violencia & 40,4 & 30,6 \\
Regiones con mucha violencia & 45,2 & 36,6 \\
\hline
\end{tabular}

Adaptado de ENDES 2000 y ENDES $2014^{(5,6)}$.

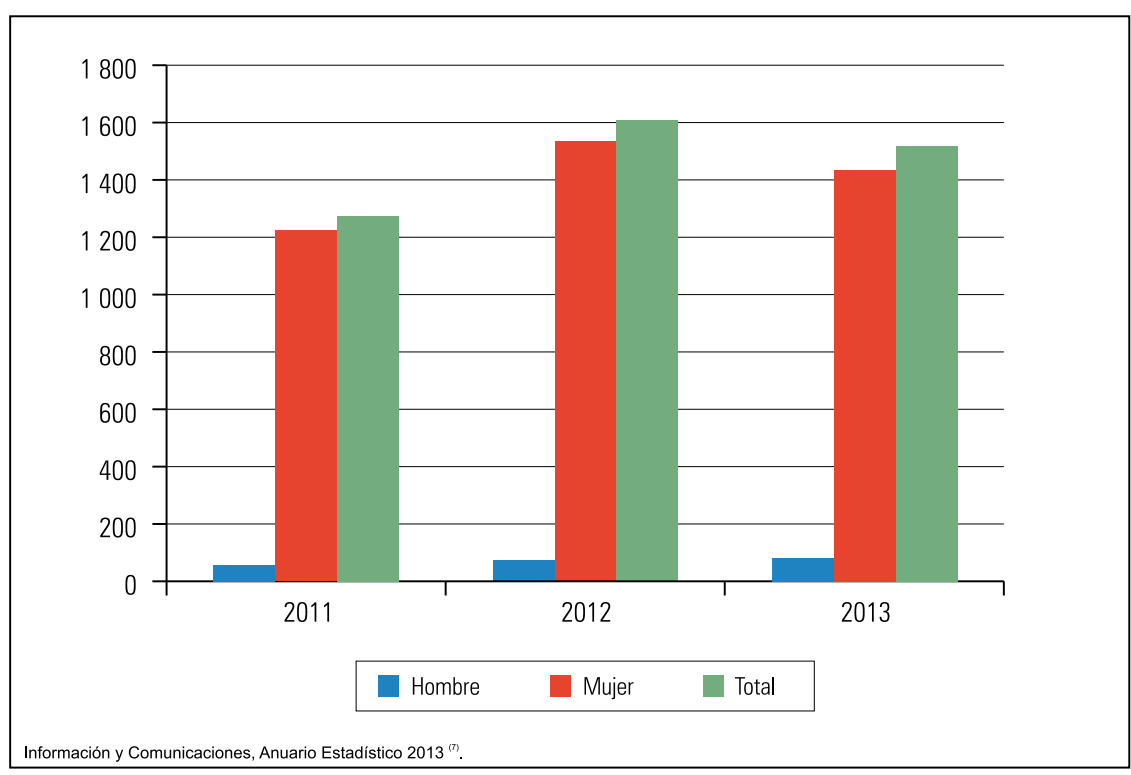

Gráfica 2. Denuncias por violencia sexual a personas mayores de 18 años.

\section{VIOLENCIA INFANTO-JUVENIL}

Existen diferentes formas de violencia contra los niños y adolescentes, la mayoría de las cuales se producen dentro del ámbito familiar, sea por acción o por omisión o negligencia. En el año 2014, se efectuaron 11097 denuncias de violencia familiar en personas de 0 a 17 años, de las cuales $57,05 \%$ fueron mujeres y $42,94 \%$ varones. Una de las formas frecuentes está relacionada a la forma de corregir de uno o ambos progenitores a las supuestas o reales faltas que cometen los hijos; generalmente son de tipo físico, pero también de carácter psicológico. Las causas y formas están vinculadas al grado de cultura, situación socio económica, relaciones entre los padres, relaciones padreshijos, relaciones entre hermanos, existen inclusive diferencias de acuerdo a las diferentes regiones del país ${ }^{(8)}$. Del mismo modo, es preocupante la alta prevalencia de abandono de los hijos por parte del padre, tanto en el aspecto afectivo como en la responsabilidad de cubrir sus necesidades de alimentación, salud, educación y recreo; existen tasas elevadas de madres solteras, inclusive adolescentes. Se pueden señalar como causas a la disfunción familiar por uniones frágiles e inestables, promiscuidad y baja autoestima de las madres. una parte importante de las violaciones no son denunciadas.

En el quinquenio 2010 a 2014 el total de feminicidios, entre los efectivizados y tentativas, se incrementaron en una forma alarmante; llegó en total a la cifra de 282 en los años 2013 y 2014 (ver gráfica 3). Las formas más utilizadas fueron golpizas, asfixia, acuchillamiento, uso de armas de fuego, aplastamiento, decapitación, quemaduras, desbarrancamiento y otras (gráfica 4).

El perpetrador en el $90 \%$ de casos fue una persona conocida o relacionada con la víctima, en la mayoría de casos la pareja o ex-pareja, que no muestra diferencias entre los años 2010 al 2014 (ver gráfica 5).

En la gráfica 6, se muestra la distribución de los feminicidios en las 10 regiones con el mayor número de casos durante el año 2014.

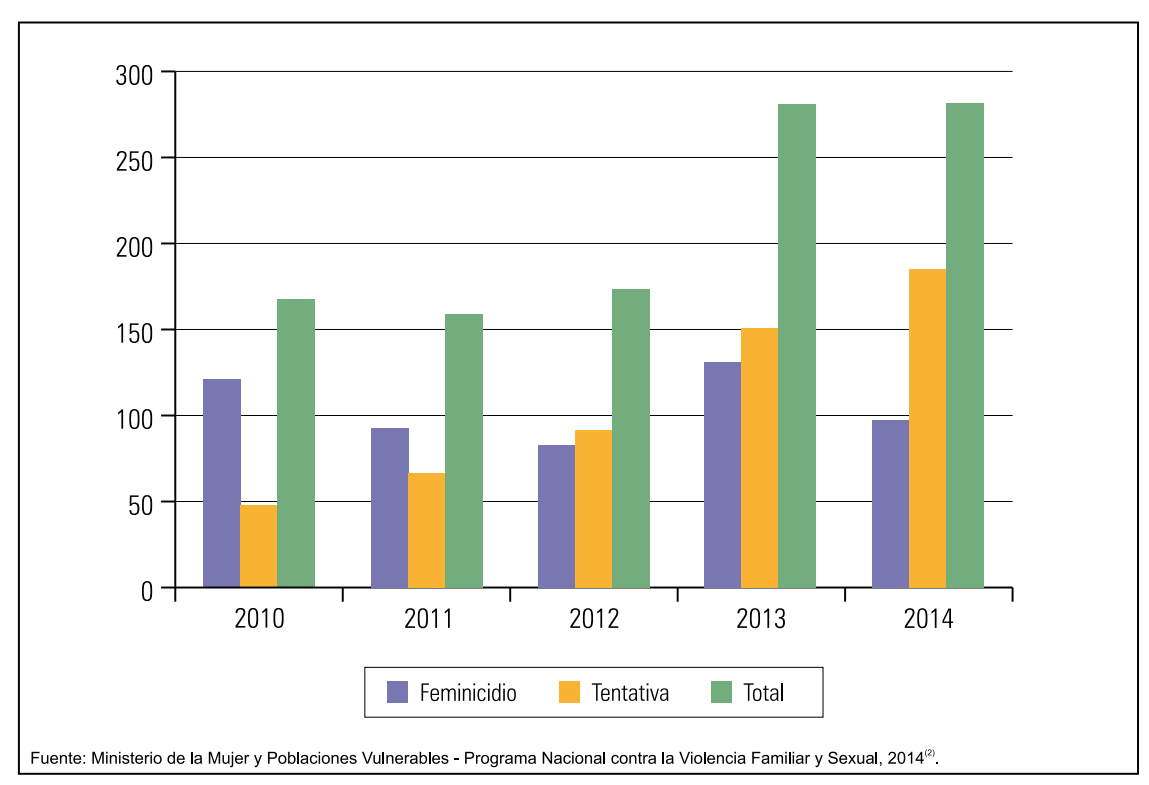

Gráfica 3. Número de feminicidios en el quinquenio 2010-2014 a nivel nacional. 


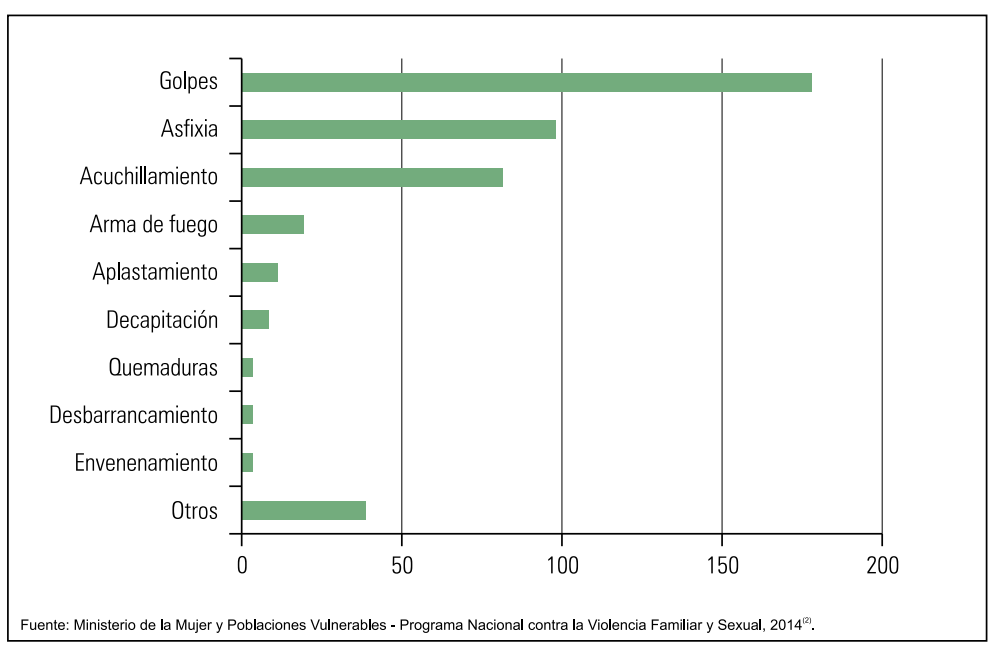

Gráfica 4. Formas de feminicidio en el año 2014.

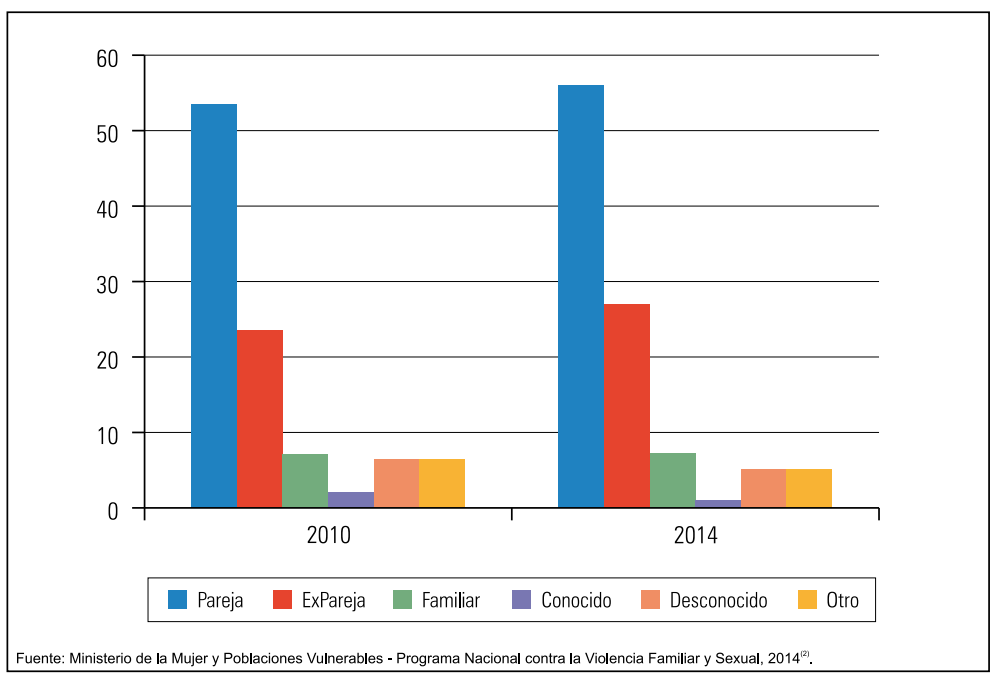

Gráfica 5. Relación porcentual entre el perpetrador y la víctima de feminicidio a nivel nacional.

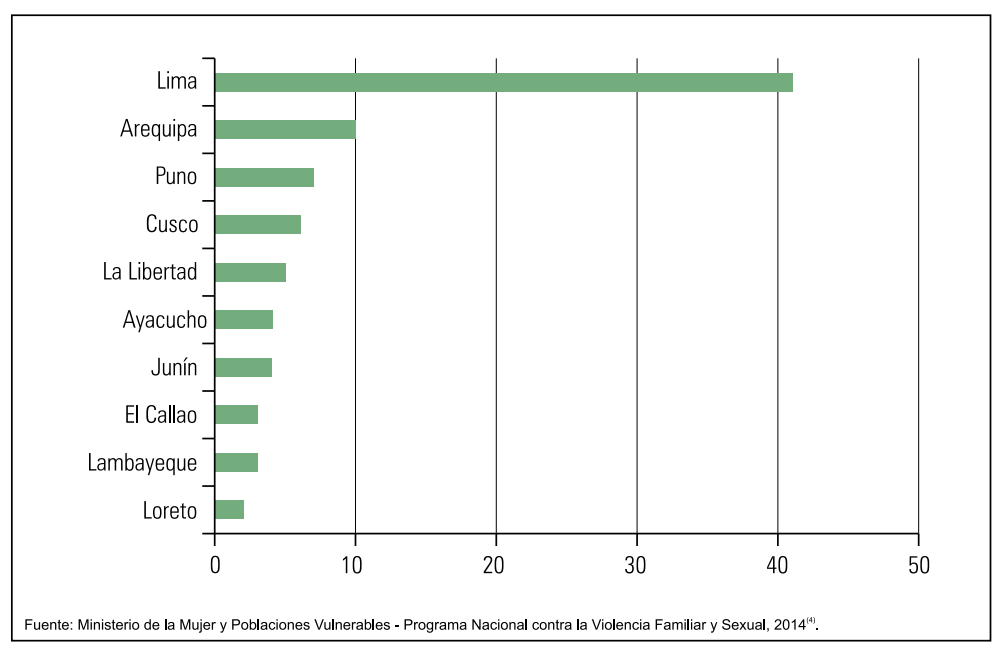

Gráfica 6. Número de feminicidios por regiones en el año 2014.
En el trabajo de Ballona y col. ${ }^{(9)}$, se informa que de 2530 niños que requirieron atención médica en el Instituto Nacional de Salud del Niño por la magnitud del trauma, 35,81\% de los cuales fue por violencia sexual, $23,95 \%$ por maltrato físico, 22,21\% por negligencia y 18,03 por violencia emocional o psicológica. Entre los años 2011 al 2013 se efectuaron entre 4000 y 4500 denuncias anuales a nivel nacional por violencia sexual en menores de 18 años, mas del $90 \%$ en niñas, tal como se puede apreciar en la gráfica 7, la mayoría de ellas registradas en la región Lima ${ }^{(7)}$.

El bullying o acoso escolar es otra de las formas de violencia desatada en contra de niños y adolescentes. Oliveros y col. han comprobado la existencia de bullying en $48 \%$ de niños encuestados en colegios estatales de Lima Este, Ayacucho, Sicuani (Cusco) y San Martín de Pangoa (Satipo, Junín) y 54,7\% en un colegio privado de Lima ciudad. En los colegios estatales el tipo de violencia fue física $34,8 \%$, verbal $34,5 \%$, psicológica $9,5 \%$ y sexual en $4,78 \%$; en el colegio privado la violencia fue verbal en $38,9 \%$, física $19,5 \%$, psicológica $3,3 \%$ y sexual $2,2 \%^{(10,11)}$.

\section{VIOLENCIA AL ADULTO MAYOR}

El maltrato psicológico a la población adulta mayor es el más frecuente en forma de insultos, humillaciones $(66,3 \%)$, amenazas de muerte $(40 \%)$, rechazo $(48,8 \%)$, maltrato físico $39 \%$, abuso sexual $2 \%$; la proporción de de mujeres/varones es de 3 a 1 ; sin embargo, en la información del año 2014 de los Centros de Ayuda Mujer de 2152 personas adultas mayores afectadas por violencia familiar $81,5 \%$ fueron mujeres ${ }^{(4,12)}$, efectuada principalmente por los hijos mayores en $44,5 \%$ de los casos, el cónyuge en $27,7 \%$, otros familiares $12 \%$, quienes entre otras formas de violencia los obligan a mendicidad, en ocasiones disimulada, como limpiar las lunas de los automóviles o la venta de golosinas. 


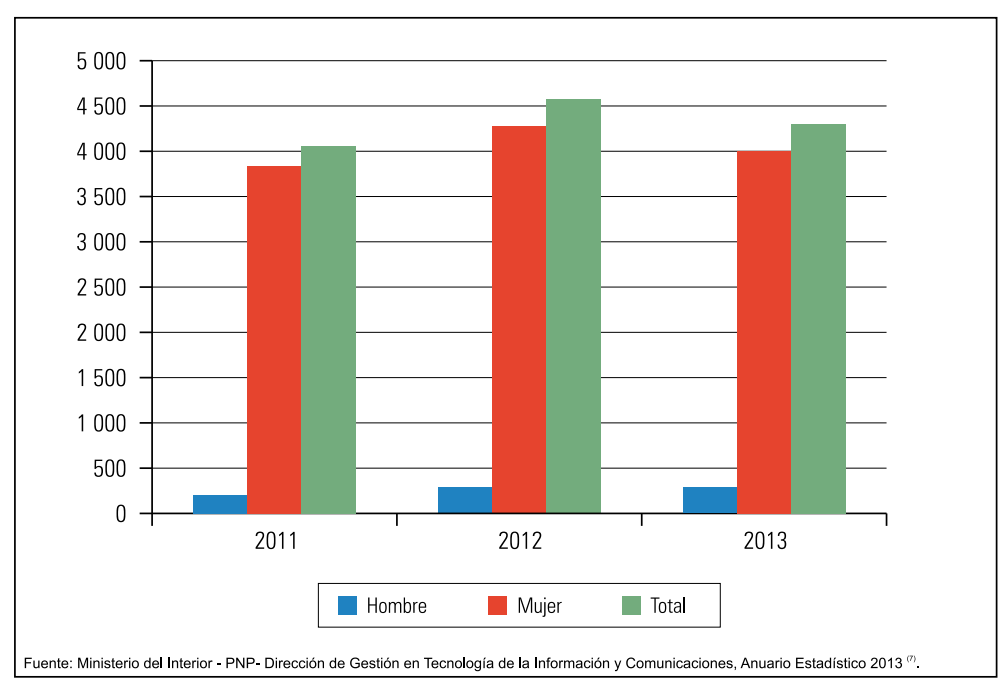

Gráfica 7. Denuncias por violencia sexual en menores de 18 años.

\section{LA VIOLENCIA EN LA DIVINA COMEDIA}

Dante Alighieri en la Divina Comedia ${ }^{(12)}$, que habría sido escrita entre los años 1300 a 1304, relata en su visita al Infierno guiado por su maestro Virgilio, que cuando llegaron al sétimo círculo encontraron recluidos a los violentos, custodiados por el Minotauro (loca bestialidad), por haber cometido diversas formas de violencia como la ejercida a otras personas (interpersonal), contra sí mismos (autoinflingida), contra Dios (blasfemos), contra la naturaleza (sodomitas) y contra el arte (usureros), que en varios aspectos coincide con la clasificación actual de la OMS mostrada en la tabla 2. Sorprende que desde esa época remota ya existiera un concepto claro sobre los tipos de violencia, lo que nos permitirá revisar las formas de violencia que han aparecido o se han intensificado en nuestro país.

La violencia contra Dios, señalada por Dante, podría ser ampliada a todas las formas de violencias de carácter religioso, violatorias de la libertad de culto, que se han producido en diferentes épocas de la historia de la humanidad, como las Cruzadas, la Santa Inquisición, la Matanza de San Bartolomé en el siglo XVI y en la actualidad a las ejercidas por Al Qaeda, el Estado Islá-
Tabla 2. Clasificación de la violencia.

1. Autoinfligida
1.1. Comportamiento suicida
1.2. Autolesiones
2. Interpersonal
2.1. Familia/Pareja
2.1.1. Menores
2.1.2. Pareja
2.1.3. Anciano
2.2. Comunidad
2.2.1. Amistades
2.2.2. Extraños
3. Colectiva
3.1. Social
3.2. Política
3.3. Económica

Informe Mundial sobre la Violencia y Salud, OMS, $2002^{(13)}$.

mico, el Boko Haram - expresiones fundamentalistas-, intolerantes de diferentes religiones, muchas de las cuales han trascendido a violencias de carácter político; a todo esto habría que añadir la proliferación de sectas que en oportunidades han conducido a esclavización, sacrificios humanos o suicidios masivos.

La violencia contra el arte se refiere a todas las formas de violencia económica, en particular a los derechos laborales; y la violencia contra la naturaleza podría hoy en día extenderse no solo a la violencia contra la libertad sexual, a la discriminación por la opción sexual, sino también a todas las modificaciones que ha impuesto la humanidad al orden natural de las cosas y que está violando los derechos humanos por afectar el bienestar, salud y vida de las personas.

\section{FORMAS EMERGENTES DE VIOLENCIA}

La revisión de la violencia en el Perú en el año 2015, nos lleva a la conclusión que se han agudizado formas que ya habían disminuido y han aparecido otras que están gravitando negativamente en nuestra sociedad actual que, en términos generales, las podemos resumir en la corrupción, la inseguridad ciudadana y el cambio climático; en una forma más desarrollada las encontramos en la tabla 3.

Entre las diferentes formas de violencia de carácter económico, en primer lugar está la corrupción. En la historia del Perú se pueden recopilar muchos hechos de corrupción de las altas esferas del gobierno. Pero, en los últimos años este tipo de violencia económica se ha incrementado, basta con recordar

Tabla 3. Formas emergentes y reemergentes de la violencia en el Perú 2015.

\begin{tabular}{l}
\hline Económica \\
Corrupción \\
Evasión tributaria \\
Delincuencial \\
Narcoterrorismo \\
Extorsión \\
Sicariato \\
Trata de personas \\
Invasión de tierras \\
Laboral \\
Sindicatos \\
Accidentes de tránsito \\
Ecológica \\
Calentamiento global \\
Minería ilegal \\
Tala de los bosques \\
Producción de biocombustibles, biodiesel y \\
y abonos de fuentes alimenticias \\
\hline
\end{tabular}




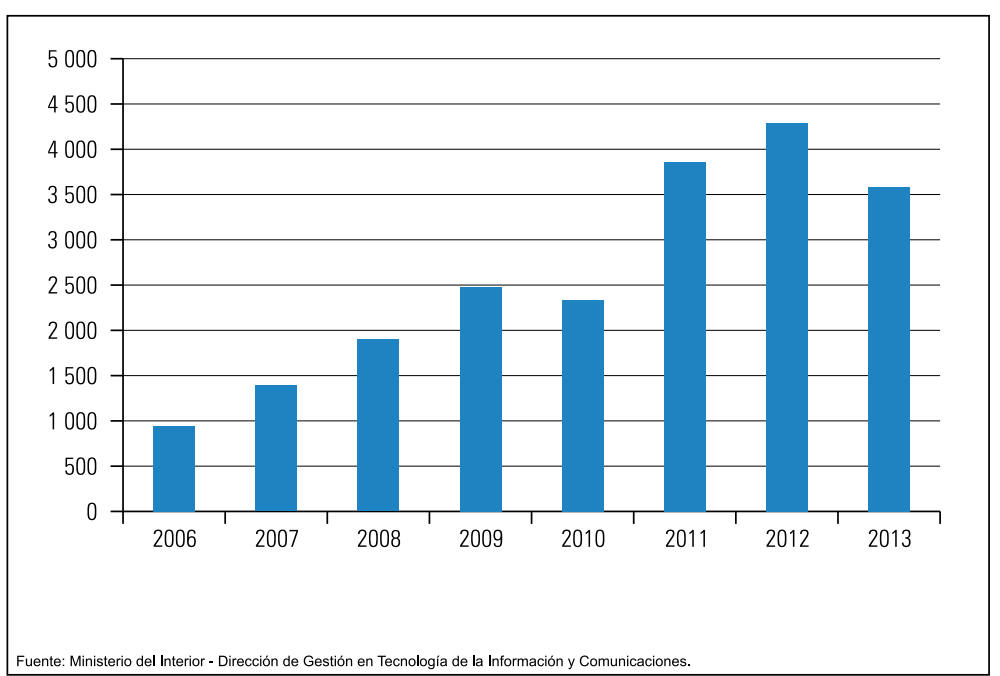

Gráfica 8. Bandas delincuenciales desbaratadas a nivel nacional.

al número importante de gobernadores, antes denominados presidentes regionales, alcaldes, magistrados, políticos, asesores, inclusive ex presidentes que se encuentran encarcelados, no habidos o cuestionados por malos manejos de los recursos asignados a su gestión. Pero la corrupción llega también a niveles de menor rango, en los que la coima para obras civiles, influencias, encubrimiento de delitos y aún de faltas, es procedimiento muy corriente. La corrupción atenta contra la economía de todos los peruanos, en especial de los más necesitados, para los cuales ese dinero mal

habido podría ser de gran utilidad o inclusive indispensable. Bajo este mismo concepto se debería considerar a la evasión tributaria que llega a los 25000 millones de soles anuales, $80 \%$ de los cuales corresponde a la efectuada por la minería y construcción informal; así mismo, se debe tener en cuenta al contrabando.

Dentro de la violencia delincuencial, llevada a cabo por personas dedicadas a la realización de actos ilícitos con fines de beneficio personal violando derechos humanos (ver gráfica 8), están los remanentes del terrorismo de

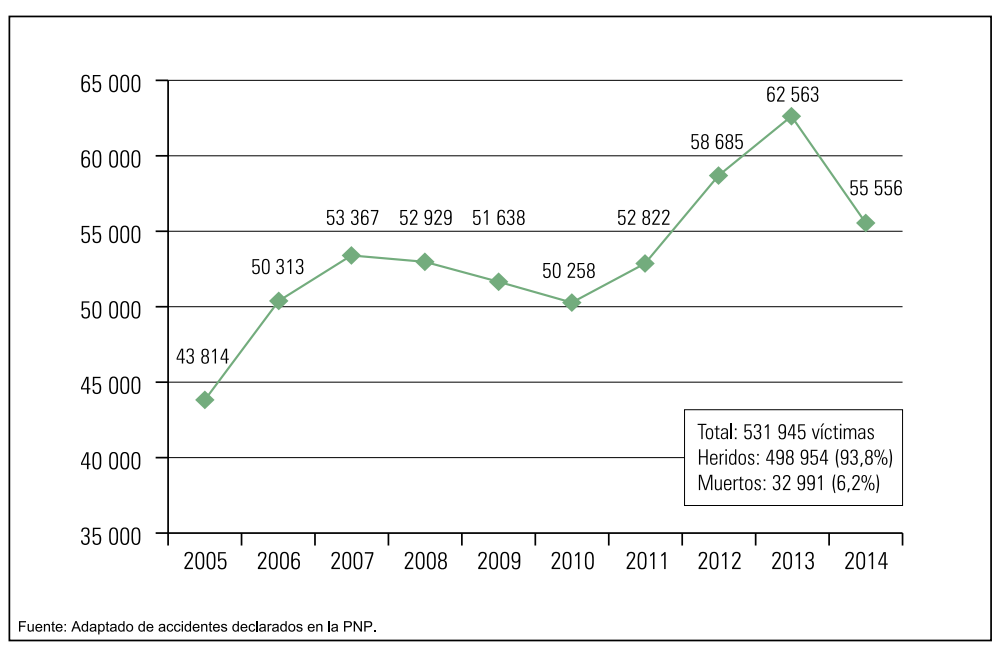

Gráfica 9. Registro de la víctimas de accidentes de tránsito. los años 1980-2000, que se han convertido en narcoterrorismo al proveer seguridad y apoyo a los narcotraficantes. El ejemplo más destacado es la situación existente en el VRAEM, donde además se esclaviza y militariza a niños. Un problema alarmante es aparición de mafias de diversa magnitud que utilizan la extorsión y el sicariato tanto en Lima, El Callao, La Libertad, Lambayeque como en Piura ${ }^{(5)}$, inclusive con el uso de granadas de mano y bombas de fabricación casera para amedrentar a personas e instituciones educativas. Se ha denunciado la modalidad de préstamos de dinero por parte de usureros colombianos con altos intereses, que extorsionan violentamente para el cobro de los préstamos. La trata de personas es la explotación de personas con fines económicos sin su consentimiento, mediante el engaño, la amenaza y el uso de la fuerza; la forma más frecuente es la explotación sexual de mujeres que puede llegar a la esclavitud. De acuerdo al Observatorio de Criminalidad del Ministerio Público ${ }^{(14)}$, en el año 2013 se efectuaron 803 denuncias, 596 fueron mujeres (74,2\%), 161 varones $(20 \%)$ y no hubo suficiente información en 46 (5,7\%). El 53,9\% de los casos debidamente establecidos correspondió a personas menores de 17 años. Los factores que facilitan este tipo de delito son la vulnerabilidad de las personas por inexperiencia, poca educación y escasos recursos económicos, la impunidad por debilidad del Poder Judicial, la informalidad del sistema social, escasa ayuda del Estado para combatir este problema y el hecho que este tipo de agresión social es muy lucrativo; a nivel global se lo ubica en el tercer lugar después del narcotráfico y el tráfico de armas. Otra modalidad que vulnera la economía de las personas es la practicada por mafias dedicadas a la invasión de terrenos y tierras de propiedad estatal y privada, que las ofrecen para la construcción de viviendas a personas que muchas veces son estafadas bajo esa modalidad.

Bajo el concepto de violencia de tipo laboral se debe considerar al maltrato que existe en los centros laborales, in- 
cluyendo a los de salud, a los sindicatos de accionar violento y a empresas con actuación negligente que ponen en riesgo la salud, vida y economía de los usuarios. En el sindicato de construcción, con el boom de la construcción, han proliferado las mafias o pseudo sindicatos que cobran cupos a los trabajadores y a los constructores, han llegado inclusive a las luchas de poder con la implementación del sicariato y los homicidios descritos como ajustes de cuentas. Otro problema laboral es el relativo al transporte terrestre de pasajeros; entre el año $2005 \mathrm{al} 2014$ se registró 531945 víctimas de accidentes de tránsito con un saldo de 498954 heridos (93,8\%) y 32991 muertos $(6,2 \%)^{(15)}$ (ver gráfica 9). Se ha demostrado que los conductores no duermen ni descansan el número suficiente de horas, lo que los pone en riesgo de provocar accidentes; en 24\% de choferes encuestados se estableció que habían tenido accidentes o estuvieron a punto de provocarlos ${ }^{(16)}$, constituyendo violencia por negligencia, cometida tanto por las empresas de transportes como de los propios conductores, que en muchos casos constituyen homicidios culposos.

La violencia contra la naturaleza señalada por Dante Alighieri, por un lado debe ser reorientada a la violencia contra la libertad sexual y a la discriminación por la opción sexual y, por otro, a todos los grandes cambios que ha producido el hombre al medio ambiente desde el inicio de la revolución industrial y que violan los derechos humanos al afectar el bienestar, salud y vida de las personas debido al calentamiento global y contaminación ambiental, que están trayendo enfermedades y muertes de diversa naturaleza, inundaciones, avalanchas, sequías. Los gobernantes de los países más industrializados tienen una gran responsabilidad, al ser conscientes de todos estos problemas y no tomar medidas efectivas para solucionarlos. A este tipo de violencia contribuyen la tala indiscriminada de los bosques, la minería ilegal como la que persiste en Madre de Dios. Un aspecto que pasa desapercibido es la producción de los denominados agrocombustibles, cuando las áreas de cultivo para la alimentación humana como el maíz, trigo, azúcar, aceite de palma son convertidas a áreas para la producción de combustibles a base del alcohol y para los biodiesel. El Perú es uno de los mayores productores de harina de pescado a base de la pesca de la anchoveta y otras especies marinas que se utiliza como abono, cuando debería estar destinada al consumo humano, frente a la existencia de grandes poblaciones en condición de pobreza y extrema pobreza que sufren de hambre y desnutrición.

\section{PROPUESTA DE UNA NUEVA CLASIFICACIÓN DE LA VIOLENCIA}

Por las consideraciones anteriores, somos de la opinión que se debería efectuar una modificación de la clasificación de los diferentes tipos de violencia a la siguiente:

Tabla 4. Propuesta para una nueva clasificación de la violencia.

1. Autoinfligida
1.1. Comportamiento suicida
1.2. Autolesiones
2. Interpersonal
2.1. Familia
2.1.1. Menores
2.1.2. Pareja
2.1.3. Anciano
2.2. Amistades
2.3. Extraños
3. Comunidad
3.1. Escolar
3.2. Laboral
3.3. Vecinal
4. Colectiva
4.1. Social
4.1.1. Corrupción
4.1.2. Delincuencial
4.2. Política
4.3. Religiosa
4.4. Económica
4.5. Ecológica

\section{LA ETIOPATOGENIA DE LA VIOLENCIA}

Al recordar el antiguo pasaje bíblico de la muerte de Abel por manos de su hermano Caín, nos hace considerar que la violencia es tan antigua como la historia de la humanidad y que podría ser considerada como una característica innata de la especie humana, cuyas causas han sido sistematizadas en el interesante Modelo Ecológico de Urie Bronfenbrenner ${ }^{(17)}$, para explicar la génesis de la violencia ejercida por el género humano mediante la consideración de 4 niveles. Ver gráfica 10.

En el nivel individual se debe considerar los factores biológicos, innatos y adquiridos en la etapa del desarrollo psicosomático. En teoría, se podría atribuir a que el proceso de la evolución del hombre desde los albores de la humanidad y debido a los cambios marcados del medio ambiente le han impreso genéticamente conductas orientadas a la sobrevivencia y la competitividad, a lo que se deben agregan disturbios del desarrollo psicosomático, innatos o adquiridos, que se exteriorizan por rasgos de impulsividad y codicia, bajo nivel educativo, comportamiento agresivo o de haber sufrido maltrato, abuso de sustancias psicotrópicas, falta o poco desarrollo de los valores morales. En este nivel del modelo ecológico se centra la atención en las características del individuo que aumentan la probabilidad de ser perpetrador o víctima de actos de violencia.

En el segundo nivel el modelo indaga la naturaleza de las relaciones sociales cercanas, en la familia, la escuela y el trabajo. En la familia, las relaciones entre los padres, entre padres e hijos y entre hermanos son muy importantes; en las familias disfuncionales, en las cuales se producen casos de la violencia infligida por la pareja y del maltrato de los hijos, aumenta el riesgo de convertirse en víctima o perpetradores de actos violentos. El acoso escolar o bullying es una de las formas más frecuentes de violencia en la escuela, que puede ser física, sexual, psicológica 


\section{ETIOPATOGENIA DE LA VIOLENCIA Modelo Ecológico}

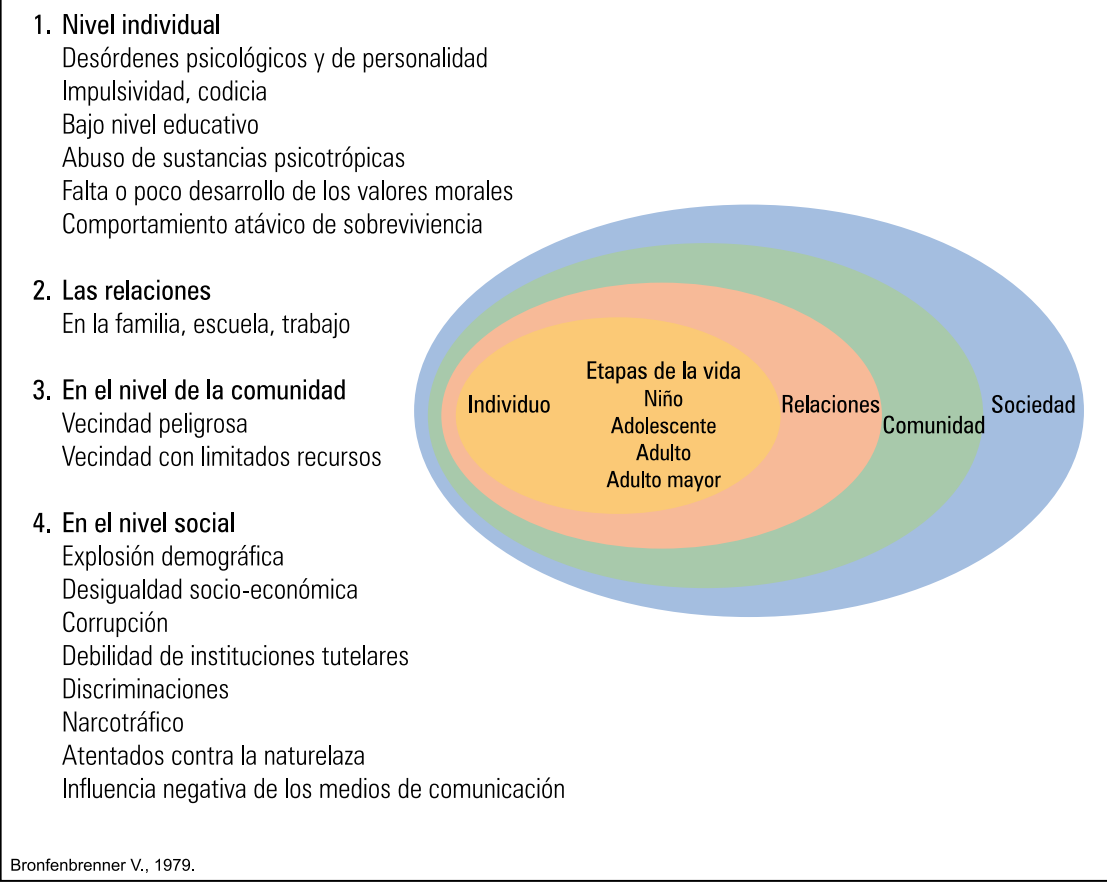

Gráfica 10. Adaptación del Modelo Ecológico a la explicación de la violencia.

(amenazas, humillaciones, discriminaciones, despojo o hurto) no solo directa sino inclusive por internet, puede ser individual o en grupos; las relaciones con amigos violentos o con conducta delincuencial puede convertir al niño o adolescente en víctima o perpetrador de violencia. Las relaciones en el trabajo no siempre son buenas, tanto entre patrones o funcionarios y trabajadores así como entre trabajadores entre sí. El acoso sexual es una forma, así como los actos de abuso de autoridad o de desconocimiento de las jerarquías laborales. En todos estos casos, la interacción casi diaria o el compartir el espacio vital con un agresor pueden aumentar las oportunidades para que se produzcan encuentros violentos.

El tercer nivel examina las interrelaciones de los miembros de la comunidad, particularmente en el vecindario. Se ha señalado como factores de riesgo a la frecuente movilidad de residencia o domicilio, la heterogeneidad (po- blación sumamente diversa, con una escasa o nula cohesión social), una densidad de población alta, vecindarios peligrosos, comprometidos con el tráfico de drogas, el desempleo elevado o el aislamiento social generalizado (cuando las personas no conocen a sus vecinos o no tienen ninguna participación en las actividades locales), las zonas de pobreza o deterioro físico, o donde hay poco apoyo institucional. Se debe agregar a la proliferación del pandillaje que involucra a núcleos de jóvenes con diferentes perspectivas, inclusive de actividades deportivas como la formación de las denominadas barras bravas que no se limitan a la agresión verbal ni física sino se llega al homicidio.

El cuarto nivel examina los factores sociales más generales que facilitan las tasas de violencia, entre los que se debe incluir a la explosión demográfica, desigualdad socio-económica, corrupción, debilidad de las instituciones tutelares (poder judicial, policía), las discrimina- ciones (de género, social, económica, étnica, de la opción sexual, de la posición de enfermedades como las mentales, TBC, sida), narcotráfico, tráfico de armas y se debe incluir a los atentados contra la naturaleza (calentamiento global, contaminación ambiental, tala indiscriminada de los bosques, minería informal e ilegal, utilización de alimentos para la producción de biocombustibles o fertilizantes en vez de su uso para la nutrición humana, tomando en consideración que existen segmentos de la población en situación de pobreza y extrema pobreza, y la influencia de los medios de comunicación que sensibilizan morbosamente diversas expresiones de violencia local y global.

\section{PROPUESTA DE ALGUNAS MEDIDAS PARA DISMINUIR LA VIOLENCIA}

La erradicación de la violencia es realmente un mito; sin embargo, se deben tomar medidas que disminuyan las tasas e intensidad de todas las formas de violencia. Entre ellas, se debe promover la educación basada en la Ética del Cuidado del prójimo ${ }^{(18)}$ y del entorno; se debe efectuar esfuerzos para disminuir las tasas de natalidad y procurar una mayor desconcentración de la población, desarrollo de políticas para una mejor distribución de la riqueza; se debe contar con un poder judicial probo y célere que evite la impunidad, desarrollo de los valores morales desde una edad temprana, educación cívica para conocer y practicar el respeto a los derechos humanos y la sobriedad en las costumbres que morigere la codicia del enriquecimiento rápido, desigual e ilícito.

Desarrollo de los valores morales ancestrales (ama sua, ama quella, ama llulla, Ayni, Minka, Ayllu, Mit'a). Desarrollo e Inclusión social mediante la música (Shinichi Suzuki, Sinfonía por el Perú, Música para Transformar Vidas). El autor del método Suzuki dijo "La enseñanza de música no es mi propósito principal. Deseo formar a buenos ciudadanos, seres humanos nobles. Si 
un niño oye buena música desde el día de su nacimiento, y aprende a tocarla él mismo, desarrolla su sensibilidad, y disciplina y paciencia. Adquiere un corazón hermoso". Juan Diego Flores tenor lírico ha manifestado "La música tiene la capacidad de transformar, de generar riqueza espiritual allí donde hay pobreza material, de empoderar y dar esperanza. El niño al sentirse parte de una orquesta o un coro se muestra con nobleza hacia su comunidad, es apreciado, felicitado y reconocido" ${ }^{(19)}$.

Se debe incentivar el reconocimiento de los paradigmas de la lucha contra la violencia, como Nelson Mandela que habiendo estado 29 años en la cárcel por luchar por los derechos de los nativos de Sudáfrica surgió en la política sin rencores; Martin Luther King con su famosa frase "I have a dream" el sueño de la restitución de los derechos humanos de la población afro-americana, Malala Yousafzai adolescente que luchó por los derechos de las mujeres por una apropiada educación, acuñó la frase "Un niño, un profesor, un libro y un lápiz pueden cambiar al mundo"; y Mahatma Gandhi que mediante la resistencia pacífica logró expulsar de la India al poderoso Imperio Británico logrando así su independencia. Justamente hoy más que nunca es necesario repetir con frecuencia sus memorables frases "Yo te ofrezco paz, yo te ofrezco amor, yo te ofrezco amistad, yo veo tu belleza, yo escucho tu necesidad, yo siento tus sentimientos, mi sabiduría fluye de una fuente superior, yo saludo en ti a esa fuente, trabajemos juntos por la unión y la paz".

\section{REFERENCIAS BIBLIOGRÁFICAS}

1. Comisión de la Verdad y Reconciliación. Informe Final, Lima, 2003.

2. Portal de la Sociedad Peruana de Prevención del Suicidio. Disponible en: http://www.spps.com.pe/ contactenos/.

3. Perales A, Sogi C. Conducta suicida en estudiantes de medicina. Rev Psiquiatr Peru. 2000;56(1):8-13.

4. Portal del Ministerio de la Mujer y Poblaciones Vulnerables - Programa Nacional contra la Violencia Familiar y Sexual, 2014.

5. Instituto Nacional de Estadistica e Informática. Perú: Encuesta Demográfica de Salud Familiar. ENDES 2000. Disponible en: www.inei.gob.pe/ biblioineipub/Est/Lib0413/Lib.

6. Instituto Nacional de Estadística e Informática. Perú: Encuesta Demográfica de Salud Familiar ENDES 2014. Disponible en: http://proyectos.inei. gob.pe/endes/2014/.

7. Portal del Ministerio del Interior - Policia Nacional del Perú, Dirección de Gestión en Tecnologia de la Información y Comunicaciones, Anuario Estadístico 2013.

8. Cano CB. Patología psiquiátrica en niños y adolescentes que habitan en zonas con antecedente de violencia social en el Perú. Diagnóstico. 2006;(4):164-9.

9. Ballona R, Chacón O, Zaldivar E y col. Manifestaciones cutáneas del maltrato Infantil. Instituto de Salud del Niño 1995 - 2002 Dermatol Pediatr Lat. 2003;1(1):24-9.

10. Oliveros M, Barrientos A. Incidencia y factores de riesgo de la intimidación (bullying) en un colegio particular de Lima-Perú, 2007. Rev per pediatr. 2007;60(3):150-5.

11. Oliveros M, Figueroa L, Mayorga G y col. Violencia escolar (bullying) en Colegios Estatales de Primaria en el Perú. Rev Soc Peruana Pediatria. 2008;61(4):215-20.
12. Lachs MS, Pillemer KA. Elder abuse. N Engl J Med. 2015 Nov 12;373(20):1947-56. doi: 10.1056/ NEJMra1404688.

13. Alighieri D. La Divina Comedia. Parte Primera, El Infierno. México: Unión Tipográfica Editorial Hispano Americana, 1961.

14. OPS/OMS Informe Mundial sobre la Violencia y Salud. Resumen, 2002.

15. Portal del Ministerio Público. Observatorio de Criminalidad. Informe deTrata de Personas, 2014.

16. Portal del Ministerios de Transportes y Comunicaciones/ Informes y Publicaciones/ Estadísticas/ Transportes/Servicios/Accidentes/Victimas de accidentes de Tránsito Fatales y no Fatales, según Características de Las Victimas: 2005-2014.

17. Caso A, Rey de Castro J, Rosales-Mayor E. Hábitos de sueño y accidentes de tránsito en conductores de omnibus interprovinciales de Arequipa, Perú. Rev Peru Med Exp Salud Publica. 2014;31(4):707-11.

18. Torrico E, Santín C, Andrés M y col. El modelo ecológico de Bronfenbrenner como marco teórico de la Psicooncologia. Anales de Psicología. 2002;18(1):45-59.

19. Weis R. Programa de Formación Ética: Desarrollo de una cultura del Cuidado. Bs As, Argentina: Ediciones Novedades Educativas del Centro de Publicaciones Educativas y Material Didáctico SRL, 2007.

20. Flores JD. El Comercio, 30 de agosto 2015.

Fuente de Financiamiento: autofinanciada.

Conflicto de interés: ninguno.

Correspondencia:

Dr. Fausto Garmendia.

Calle Elias Aguirre No 144-409, Miraflores, Lima, Perú.

Teléfonos: $981903903-2418956$

Correo electrónico: garmendiafausto@gmail.com 